Modelling sound propagation in acoustic waveguides using a hybrid numerical method

\title{
Ray Kirby
}

\section{School of Engineering and Design,}

Mechanical Engineering,

Brunel University, Uxbridge, Middlesex, UB8 3PH, UK.

ray.kirby@brunel.ac.uk 
Kirby, JASA

\begin{abstract}
Sound propagation in an acoustic waveguide is examined using a hybrid numerical technique. Here, the waveguide is assumed to be infinite in length with an arbitrary but uniform crosssection. Placed centrally within the guide is a short component section with an irregular, nonuniform, shape. The hybrid method utilises a wave based modal solution for a uniform section of the guide and, using either a mode matching or point collocation approach, matches this to a standard finite element based solution for the component section. Thus, one needs only to generate a transverse finite element mesh in uniform sections of the waveguide and this significantly reduces the number of degrees of freedom required. Moreover, utilising a wave based solution removes the need to numerically enforce a non-reflecting boundary condition at infinity using a necessarily finite mesh, which is often encountered in studies that use only the standard finite element method. Accordingly, the component transmission loss may readily be computed and predictions are presented here for three examples: an expansion chamber, a converging-diverging duct and a circular cylinder. Good agreement with analytic models is observed, and transmission loss predictions are also presented for multi-mode incident and transmitted sound fields.
\end{abstract}




\section{INTRODUCTION}

The study of non-uniform obstructions in an otherwise uniform waveguide is of fundamental interest and it is not surprising that this subject has received extensive attention in literature. Applications are many, including underwater sound propagation, the transmission of elastic and electromagnetic waves, and sound propagation in ducts or pipes. It is common in these applications for relatively long uniform sections to be present, which are punctuated by relatively short area changes and/or non-uniform obstacles. This article is concerned with the application of a general numerical method suitable for examining sound propagation in uniform acoustic waveguides of arbitrary cross-section that contain one or more non-uniform obstacles, or areachanges, placed centrally within the guide.

The method presented here will focus on sound propagation in ductwork and so is applicable to rectangular ventilation ducts but also to circular and oval ductwork. The key challenge here is to model accurately the scattering of sound waves from non-uniform area discontinuities in relatively large ductwork. Of course, the study of sound scattering from area discontinuities goes back to Rayleigh, although traditionally it has been possible only to examine relatively simple non-uniform geometries with modest dimensions. More recent examples include Miles ${ }^{1}$, and Selamet and Easwaran ${ }^{2}$, who examined plane wave propagation in variable area ducts; and Boström $^{3}$ who used analytic techniques to study scattering by spherical and spheroidal obstacles in a duct. Boström examined ducts of arbitrary cross-section but noted that tractable solutions were possible only for a limited range of geometries. The study of sound scattering from obstacles has also received extensive attention in the location of so-called trapped modes, see for 
example Refs. 4-7. Trapped modes are acoustic resonances near obstacles in ducts and have been found to exist for a number of geometries including parallel plates, rectangular obstructions, cylinders, and ball-type valves. The study of trapped modes is, however, largely based on analytic work and, whilst some relatively complicated obstructions have been examined, these techniques are not readily applicable to more complex non-uniform area changes and/or ducts of arbitrary cross-section.

The study of sound propagation over a fully arbitrary area change will inevitably require numerical methods that can cope with irregular geometries and/or step changes in boundary conditions. Suitable numerical methods include the standard finite element method (FEM) and the boundary element method (BEM). For example, Tang and Lau ${ }^{8}$, and later Lau and Tang9 used the standard FEM to study tapered and convergent-divergent sections in rectangular ducts, although both studies required a large number of degrees of freedom in order to obtain a converged solution. Jeong et al. ${ }^{10}$ reviewed the application of the BEM in rectangular ductwork and demonstrated that by discretising the duct into multiple domains one may generate an efficient BEM algorithm that may be applied to larger ductwork. However, area changes were not examined by Jeong et al. ${ }^{10}$ and it is evident that, even for relatively low frequencies, a large number of degrees of freedom were still required. In principle, numerical methods may be applied to a wide range of sound propagation problems; however, a problem that plagues numerical methods is the computational time required to obtain solutions, especially if one wishes to cover a representative frequency range for large ducts such as those commonly found in ventilation systems. Here, the FEM normally finds favour over the BEM because discretisation delivers a banded symmetric matrix ${ }^{10,11}$, although even the FEM requires a large 
number of degrees of freedom to study relatively simple problems. For example, Lau and Tang ${ }^{9}$ used a very fine mesh to study a converging-diverging duct, which has the potential to severely limit the upper frequency of the analysis as the size of the problem becomes unmanageable. A further, and arguably more fundamental, problem with numerical methods is the specification of the terminating (downstream) axial boundary condition. When studying infinite waveguides, this boundary condition should be anechoic, but problems arise when attempting to specify a boundary condition at infinity using a necessarily finite grid. Of course, for plane wave propagation one may easily represent an anechoic termination by setting the impedance of the downstream boundary to be equal to the characteristic impedance of the fluid in the duct, and this has been applied by many authors (see Refs. 9-12). However, for larger ventilation ducts, higher order modes may propagate at relatively low frequencies and this plane wave boundary condition is no longer appropriate. To overcome this Lau and Tang ${ }^{9}$ specify absorptive walls in the downstream section of their duct so that higher order modes are numerically damped down before reaching the (plane wave) terminating condition. Other methods may also be used, for example a "perfectly matched layer" or a high-order local non-reflecting boundary condition, see Givoli $^{13}$. These various non-reflecting boundary conditions have been shown to work well under certain conditions; however, these methods can be computationally expensive and may only ever provide an approximation to the desired terminating boundary condition.

An alternative numerically efficient approach is to retain a modal analysis for the uniform section and to match, or join, this analysis to a standard finite element representation for the component section. In this way, complex non-uniform sections may be studied as efficiently as possible, and so it is not surprising that this type of "hybrid" approach has been applied to a wide 
range of problems in the literature. For example, in the study of elastic waveguides, Mal and Chang ${ }^{14}$ used a hybrid method to enforce continuity conditions over discrete nodal locations that are common to the boundary between the uniform and non-uniform regions. Liu et al. ${ }^{15}$ also studied elastic waveguides but matched a finite element representation of an interior region to a Green's function integral representation of the exterior region. Other examples include Refs. 1618 , whereby the continuity conditions over the interface between two regions in electromagnetic waveguide are enforced using mode matching (MM). In acoustics, the application of the method is arguably less widespread and examples tend to favour exterior problems. For example, Kagawa et al. ${ }^{19}$ used the FEM to analyse an interior problem and then applied MM to "join" this solution to a Green's function representation of the exterior acoustic far-field. Astley and Cummings ${ }^{20}$ also used MM but they analysed sound radiation from a vibrating ventilation duct wall, using finite elements to discretise the exterior acoustic near field before coupling this to an eigenexpansion of the acoustic far field. A similar approach was also adopted by $\operatorname{Imhof}^{21}$, who studied both acoustic and elastic waveguides. A review of the application of a hybrid method to acoustic problems is discussed by $\mathrm{Astley}^{22}$, and here a close relationship between the finite element Dirichlet-to-Neumann (FE-DtN) and hybrid methods is demonstrated, provided one uses MM to enforce continuity conditions between the uniform and non-uniform sections. Furthermore, Astley ${ }^{22}$ applied this hybrid method to the study of interior as well as exterior acoustic problems, and for the interior problem sound propagation is modelled in a simple diverging duct, although results were reported only for the exterior problem. Results for a similar interior problem were, however, reported in an earlier paper by Astley and Eversman ${ }^{23}$, who analysed sound attenuation in a cosine tapered converging duct with mean flow and a locally reacting liner. 
Clearly, a hybrid numerical method is well suited to analysing sound propagation in interior problems such as ventilation ducts. Accordingly, this article will focus on applying the method of Astley $^{22}$ to the analysis of uniform ducts of arbitrary cross-section that include relatively complex component sections. Of interest here will be the relative convergence and accuracy of the method when compared to analytic results. This paper will also compare two different methods of enforcing the continuity conditions between the uniform and non-uniform sections, namely the MM method of Astley ${ }^{22}$ and a point collocation (PC) approach similar to the one described by Kirby and Lawrie. ${ }^{24}$ Accordingly, this article beings by outlining the governing equations for the problem, before describing the MM and PC methods; results are then presented for sound propagation in a duct containing an expansion chamber, a converging-diverging duct, and a cylinder. 


\section{THEORY}

The duct geometry to be analysed consists of three separate regions, as shown in Fig. 1. The inlet duct is denoted region $R_{1}$, and this is assumed to have a uniform but arbitrary cross-section. The inlet duct abuts onto a general arbitrarily shaped "component" section, which is denoted region $R_{2}$. Finally, the component section abuts onto the outlet duct, region $R_{3}$, which is assumed to have a uniform, but arbitrary, cross-section. In principle each region may be bounded by acoustically soft walls and/or wave bearing walls; moreover, each section may contain more than one (equivalent) fluid, for example, a porous material. Separating the three regions are two planes, $\mathrm{A}$ and $\mathrm{B}$, which lie perpendicular to regions $R_{l}$ and $R_{3}$, respectively. Here, the convective effects of mean flow may readily be added to the analysis, although the component section is likely to generate free shear layers downstream of an area discontinuity. Hydrodynamic modes may then be present and it is likely that this will introduce further numerical complications when matching over plane B downstream of an area discontinuity. Furthermore, accommodating nonuniform geometries in the component section will also require a detailed knowledge of the flow

patterns. As these issues are likely to complicate the analysis and detract from the main focus of this paper, mean flow is omitted from the analysis that follows.

Sound propagation in region $q(q=1,2$, or 3$)$ of the duct shown in Fig. 1 is governed by the acoustic wave equation 


$$
\frac{1}{c_{q}^{2}} \frac{\partial^{2} p_{q}^{\prime}}{\partial t^{2}}-\nabla^{2} p_{q}^{\prime}=0
$$

where $c_{q}$ is the speed of sound, $p_{q}^{\prime}$ is the acoustic pressure, and $t$ is time. The solution of this equation proceeds by using a method that is the most appropriate for the geometry in each region. Accordingly, the uniform geometry of regions $R_{1}$ and $R_{3}$ encourage a modal analysis and so the acoustic pressure is expanded as an infinite sum over the eigenmodes in each region. For the duct component, provision is made for a complicated non-uniform shape. Here, the wave equation is solved using the finite element method.

For regions $R_{1}$ and $R_{3}$, expansion of the sound pressure field, assuming a time dependence of $e^{\mathrm{i} \omega t}$ (where $\mathrm{i}=\sqrt{-1}$ and $\omega$ is the radian frequency), yields

$$
p_{1}^{\prime}(x, y, z)=\sum_{n=0}^{\infty} F_{n} \Phi_{n}(y, z) e^{-\mathrm{i} k_{1} \lambda_{n} x}+\sum_{n=0}^{\infty} A_{n} \Phi_{n}(y, z) e^{\mathrm{i} k_{1} \lambda_{n} x}
$$

and

$$
p_{3}^{\prime}(x, y, z)=\sum_{j=0}^{\infty} B_{j} \Psi_{j}(y, z) e^{-\mathrm{i} k_{3} \gamma_{j} x^{\prime}}+\sum_{j=0}^{\infty} C_{j} \Psi_{j}(y, z) e^{\mathrm{i} k_{3} \gamma_{j} x^{\prime}}
$$

Here, $F_{n}, A_{n}, B_{j}$, and $C_{j}$ are the modal amplitudes, $k_{q}=\omega / c_{q}, \lambda$ is the (dimensionless) wavenumber in region $R_{1}$, and $\gamma$ is the (dimensionless) wavenumber in region $R_{3}$. The quantities $\Phi_{n}(y, z)$ and $\Psi_{j}(y, z)$ are the transverse duct eigenfunctions in regions $R_{1}$ and $R_{3}$, respectively. In the analysis that follows it is assumed that regions $R_{l}$ and $\mathrm{R}_{3}$ have an arbitrary cross-section and so the eigenvalues and eigenvectors in regions $R_{1}$ and $R_{3}$ are found using the FEM. $^{25}$ The incident sound pressure field in region $R_{1}$ is assumed to be multi-modal, which 
requires knowledge of the modal amplitudes in the incident sound field. Here, equal modal energy density (EMED) is assumed for all propagating modes, as this is thought to best represent the sound field emanating from a fan in a ventilation system (see Kirby and Lawrie ${ }^{24}$ ). This choice may, however, readily be changed to include other relationships between the modal amplitude such as those discussed by Kirby and Lawrie, or by utilising Green's functions to replicate a point source. For EMED, this yields

$$
\left|\frac{F_{n}}{p_{0}}\right|^{2}=\frac{I_{0}}{I_{n} \sum_{m=0}^{n_{F}} \lambda_{m}}
$$

where $p_{0}$ is a reference pressure chosen here, arbitrarily, to be equal to unity. The number of modes propagating in region $R_{1}$ is denoted by $n_{F}$ (for modes that are "cut-off", $F=0$ ) and $I_{n}=\int_{\Gamma_{A}}\left|\Phi_{n}(y, z)\right|^{2} d y d z$, where $\Gamma_{A}$ denotes the surface of $R_{2}$ that lies on plane A.

For region $R_{2}$ the acoustic pressure is approximated by

$$
p_{2}^{\prime}(x, y, z)=\sum_{j=1}^{n_{2}} N_{j}(x, y, z) p_{2_{j}}
$$

where $N_{j}$ is a global trial (or shape) function for the finite element mesh, $p_{2_{j}}$ is the value of the acoustic pressure at node $j$, and $n_{2}$ is the number of nodes (or degrees of freedom) in region $R_{2}$. Expressing Eq. (5) in vector form yields 


$$
p_{2}^{\prime}(x, y, z)=\left[N_{1}(x, y, z), N_{2}(x, y, z), \ldots, N_{n_{2}}(x, y, z)\right]\left[\begin{array}{l}
p_{2_{1}} \\
p_{2_{2}} \\
p_{2_{n_{2}}}
\end{array}\right]=\mathbf{N p}_{2}
$$

A weighted residual statement of the wave equation may now be formulated and, after application of Green's theorem, this yields

$$
\left[\int_{\Omega_{2}}\left[\nabla \mathbf{N}^{T} \cdot \nabla \mathbf{N}-k_{2}^{2} \mathbf{N}^{T} \mathbf{N}\right] d \Omega_{2}\right]\left\{\mathbf{p}_{2}\right\}=\int_{\Gamma_{2}}\left[\mathbf{N}^{T} \nabla p_{2}^{\prime} \cdot \mathbf{n}_{2}\right] d \Gamma_{2} .
$$

Here, $\Omega_{2}$ and $\Gamma_{2}$ denote the volume and the outer surface of region $R_{2}$, respectively, and $\mathbf{n}_{2}$ is the outward unit normal vector for the surface of region 2. In order to simplify the presentation of the method, Eq. (7) assumes that a single homogenous fluid is present in region $R_{2}$; however, more than one fluid may readily be added simply by changing the wavenumber and writing a separate equation for each fluid. It is convenient here to separate planes A and B from the surface integral on the right hand side of Eq. (7) and to write

$$
\int_{\Gamma_{2}}\left[\mathbf{N}^{T} \nabla p_{2}^{\prime} \cdot \mathbf{n}_{2}\right] d \Gamma_{2}=\int_{\Gamma_{A}}\left[\mathbf{N}^{T} \nabla p_{2}^{\prime} \cdot \mathbf{n}_{A}\right] d \Gamma_{A}+\int_{\Gamma_{B}}\left[\mathbf{N}^{T} \nabla p_{2}^{\prime} \cdot \mathbf{n}_{B}\right] d \Gamma_{B}+\int_{\Gamma_{e}}\left[\mathbf{N}^{T} \nabla p_{2}^{\prime} \cdot \mathbf{n}_{e}\right] d \Gamma_{e},
$$

where $\Gamma_{A}$ and $\Gamma_{B}$ denote the surface of $R_{2}$ that lies on planes $\mathrm{A}$ and $\mathrm{B}$, respectively, and $\Gamma_{e}$ is the surface of region $R_{2}$ that does not lie on $\Gamma_{A}$ and $\Gamma_{B}$. Here, $\mathbf{n}_{q}$ is the outward unit normal vector over surface $q$.

The analysis proceeds by enforcing continuity of acoustic pressure and axial velocity over surfaces $\Gamma_{A}$ and $\Gamma_{B}$. Two different approaches to enforce these continuity conditions are examined here: the MM method of $\mathrm{Astley}^{22}$, and the PC method of Kirby and Lawrie ${ }^{24}$. For the 
MM method the number of modes utilised in regions $R_{1}$ and $R_{3}$ does not necessarily have to equal the number of nodes on $\Gamma_{A}$ and $\Gamma_{B}$, whereas the number of collocation points should equal the number of nodes on $\Gamma_{A}$ and $\Gamma_{B}$. Therefore, one would expect MM to be the more efficient of the two methods, although for interior problems it is likely that the number of nodes on $\Gamma_{A}$ and $\Gamma_{B}$ will be significantly smaller than the total number of nodes used to mesh region $R_{2}$. Hence, for interior problems the potential reduction in problem size afforded by the MM method is likely to be limited when compared to the PC method. However, Astley ${ }^{22}$ notes that, provided one uses an appropriate weighting function, the MM method is capable of delivering a symmetric stiffness matrix, which is not the case for PC. Thus, the MM method will be faster than the PC method; however the point collocation method is retained here in order to provide an alternative method that may prove useful, at least as a benchmark for the MM method.

The application of the continuity conditions and the solution of the problem will be covered in the next two sections. On solving the problem, the sound transmission loss (TL) of the component section is readily calculated from the ratio of the transmitted to incident sound powers, which yields

$$
T L=-10 \log _{10} \frac{\rho_{1} k_{3}}{\rho_{3} k_{1}} \sum_{m=0}^{n_{B}} \frac{\gamma_{m} H_{m}\left|B_{m}\right|^{2}}{I_{0}},
$$

where $H_{m}=\int_{\Gamma_{B}}\left|\Psi_{m}(y, z)\right|^{2} d y d z, n_{B}$ is the number of modes cut on in region $R_{3}$, and $\rho_{q}$ is the density of the fluid is region $q$. 
Kirby, JASA

\section{A. Mode matching}

The MM method relies on weighting the appropriate continuity conditions and then integrating over $\Gamma_{A}$ and $\Gamma_{B}$. Accordingly, making use of Eqs. (2) and (3) allows continuity of pressure and velocity over $\Gamma_{A}$ to be written as

$$
p_{2}^{\prime}(0, y, z)=\sum_{n=0}^{\infty} F_{n} \Phi_{n}(y, z)+\sum_{n=0}^{\infty} A_{n} \Phi_{n}(y, z)
$$

and

$$
\frac{\partial p_{2}^{\prime}}{\partial x}(0, y, z)=i k_{1} \beta_{1}\left\{\sum_{n=0}^{\infty} F_{n} \lambda_{n} \Phi_{n}(y, z)-\sum_{n=0}^{\infty} A_{n} \lambda_{n} \Phi_{n}(y, z)\right\} .
$$

Similarly, over $\Gamma_{B}$

$$
p_{2}^{\prime}(L, y, z)=\sum_{j=0}^{\infty} B_{j} \Psi_{j}(y, z)
$$

and

$$
\frac{\partial p_{2}^{\prime}}{\partial x}(L, y, z)=-i k_{3} \beta_{3}\left\{\sum_{j=0}^{\infty} B_{j} \gamma_{j} \Psi_{j}(y, z)-\sum_{j=0}^{\infty} C_{j} \gamma_{j} \Psi_{j}(y, z)\right\}
$$

Here, $L$ is the axial distance between $\Gamma_{A}$ and $\Gamma_{B}$, and $\beta_{q}=\rho_{2} / \rho_{q}$. To obtain a solution, it is necessary to ascribe boundary conditions to the outer surface of the problem domain. Here, complex boundary conditions such as acoustically soft or wave-bearing walls may be applied; however, in order to validate the method against analytic results the analysis that follows will assume that all outer duct surfaces are acoustically hard. Thus, for $\Gamma_{e}, \nabla p_{2}^{\prime} \cdot \mathbf{n}_{e}=0$, and for regions $R_{1}$ and $R_{3}$ the problem reduces to computing the eigenvalues and eigenvectors in a hard walled duct of arbitrary cross section. In addition, a non-reflecting boundary condition is specified in region $R_{3}$ by setting $C_{j}$ equal to zero. The velocity matching conditions, given by 
Eqs. (11) and (13), may now substituted into Eq. (8), which yields (after dropping the summations for clarity)

$$
\int_{\Gamma_{2}}\left[\mathbf{N}^{T} \nabla p_{2}^{\prime} \cdot \mathbf{n}_{2}\right] d \Gamma_{2}=i k_{1} \beta_{1} \lambda_{n} \int_{\Gamma_{A}} \mathbf{N}^{T}\left[F_{n} \Phi_{n}-A_{n} \Phi_{n}\right] d \Gamma_{A}-i k_{3} \beta_{3} \gamma_{j} \int_{\Gamma_{B}} \mathbf{N}^{T}\left[B_{j} \Psi_{j}\right] d \Gamma_{B} .
$$

The MM method of Astley $^{22}$ proceeds by weighting each pressure condition using the incident velocity in the axial direction in region $R_{1}$ (or region $R_{3}$ ) and then integrating over $\Gamma_{A}$ (or $\Gamma_{B}$ ). Thus, for $\Gamma_{A}$ Eq. (10) yields

$$
i k_{1} \beta_{1}\left\{\lambda_{m} \int_{\Gamma_{A}} \Phi_{m} \Phi_{n} d \Gamma_{A}\right\}\left\{F_{n}\right\}+i k_{1} \beta_{1}\left\{\lambda_{m} \int_{\Gamma_{A}} \Phi_{m} \Phi_{n} d \Gamma_{A}\right\}\left\{A_{n}\right\}=i k_{1} \beta_{1}\left\{\lambda_{m} \int_{\Gamma_{A}} \Phi_{m} \mathbf{N} d \Gamma_{A}\right\}\left\{\mathbf{p}_{2 A}\right\}
$$

and for $\Gamma_{B}$, Eq. (12) yields

$$
i k_{3} \beta_{3}\left\{\gamma_{m} \int_{\Gamma_{B}} \Psi_{m} \Psi_{n} d \Gamma_{B}\right\}\left\{B_{n}\right\}=i k_{3} \beta_{3}\left\{\gamma_{m} \int_{\Gamma_{B}} \Psi_{m} \mathbf{N} d \Gamma_{B}\right\}\left\{\mathbf{p}_{2 B}\right\}
$$

Here, the summation signs have again been dropped for clarity, and on use of Eq. (6), vectors $\mathbf{p}_{2 A}$ and $\mathbf{p}_{2 B}$ hold values of the finite element solution in region $R_{2}$ at the nodal locations on the surfaces $\Gamma_{A}$ and $\Gamma_{B}$, respectively. Finally, before solving it is necessary first to truncate the infinite sums, and here $m_{1}$ and $m_{3}$ will denote the number of modes assumed to be present in regions $R_{1}$ and $R_{3}$, respectively. It is then convenient to re-write the problem in matrix form, where

$$
[\mathbf{G}]=\int_{\Omega_{2}}\left[\nabla \mathbf{N}^{T} \cdot \nabla \mathbf{N}-k_{2}^{2} \mathbf{N}^{T} \mathbf{N}\right] d \Omega_{2}
$$


Kirby, JASA

$$
\begin{gathered}
{[\mathbf{Q}]=i k_{1} \beta_{1} \sum_{m=0}^{m_{1}} \lambda_{m} \int_{\Gamma_{A}} \Phi_{m} \mathbf{N} d \Gamma_{A},} \\
{[\mathbf{R}]=i k_{3} \beta_{3} \sum_{m=0}^{m_{3}} \gamma_{m} \int_{\Gamma_{B}} \Psi_{m} \mathbf{N} d \Gamma_{B},} \\
{\left[\mathbf{M}_{1}\right]=i k_{1} \beta_{1} \sum_{m=0}^{m_{1}} \sum_{n=0}^{m_{1}} \lambda_{m} \int_{\Gamma_{A}} \Phi_{m} \Phi_{n} d \Gamma_{A},} \\
{\left[\mathbf{M}_{2}\right]=i k_{3} \beta_{3} \sum_{m=0}^{m_{3}} \sum_{n=0}^{m_{3}} \gamma_{m} \int_{\Gamma_{B}} \Psi_{m} \Psi_{n} d \Gamma_{B} .}
\end{gathered}
$$

In addition,

$$
[\widetilde{\mathbf{Q}}]=i k_{1} \beta_{1} \sum_{m=0}^{m_{1}} \sum_{n=0}^{n_{F}} F_{n} \lambda_{m} \int_{\Gamma_{A}} \Phi_{m} \mathbf{N} d \Gamma_{A}
$$

and

$$
\left[\tilde{\mathbf{M}}_{1}\right]=i k_{1} \beta_{1} \sum_{m=0}^{m_{1}} \sum_{n=0}^{n_{F}} F_{n} \lambda_{m} \int_{\Gamma_{A}} \Phi_{m} \Phi_{n} d \Gamma_{A}
$$

Equations (7), (15), and (16) may now be written in matrix form:

$$
\begin{gathered}
{[\mathbf{G}]\left\{\mathbf{p}_{2}\right\}+[\mathbf{Q}]^{T}\{\mathbf{A}\}+[\mathbf{R}]^{T}\{\mathbf{B}\}=[\tilde{\mathbf{Q}}]^{T},} \\
-\left[\mathbf{M}_{1}\right]\{\mathbf{A}\}+[\mathbf{Q}]\left\{\mathbf{p}_{2 A}\right\}=\left[\tilde{\mathbf{M}}_{1}\right], \\
-\left[\mathbf{M}_{2}\right]\{\mathbf{B}\}+[\mathbf{R}]\left\{\mathbf{p}_{2 B}\right\}=\mathbf{0} .
\end{gathered}
$$

To combine all three equations, matrix $\mathbf{G}$ is decomposed into separate elements to give

$$
[\mathbf{G}]\left\{\mathbf{p}_{2}\right\}=\left[\begin{array}{lll}
\mathbf{G}_{11} & \mathbf{G}_{1 e} & \mathbf{G}_{13} \\
\mathbf{G}_{e 1} & \mathbf{G}_{e e} & \mathbf{G}_{e 3} \\
\mathbf{G}_{31} & \mathbf{G}_{3 e} & \mathbf{G}_{33}
\end{array}\right]\left\{\begin{array}{l}
\mathbf{p}_{2 A} \\
\mathbf{p}_{2 e} \\
\mathbf{p}_{2 B}
\end{array}\right\},
$$


where matrix $\mathbf{G}_{m n}$ has order $n_{m} \times n_{n}$. Here, $n_{1}$ and $n_{3}$ denote the number of nodes on $\Gamma_{A}$ and $\Gamma_{B}$, respectively (where $m_{1} \leq n_{1}$ and $m_{3} \leq n_{3}$ ); $n_{2}$ is the number of nodes in region $R_{2}$; and $n_{e}$ is the number of nodes that lie in region 2 but do not lie on $\Gamma_{A}$ and $\Gamma_{B}$ (so that $n_{e}=n_{2}-n_{1}-n_{3}$ ). The values for pressure at those nodes in region $R_{2}$ that do not lie on $\Gamma_{A}$ and $\Gamma_{B}$ are held in matrix $\mathbf{p}_{2 e}$. The problem may now be written as

$$
\left[\begin{array}{ccccc}
-\mathbf{M}_{1} & \mathbf{Q} & \mathbf{0} & \mathbf{0} & \mathbf{0} \\
\mathbf{Q}^{T} & \mathbf{G}_{11} & \mathbf{G}_{1 e} & \mathbf{G}_{13} & \mathbf{0} \\
\mathbf{0} & \mathbf{G}_{e 1} & \mathbf{G}_{e e} & \mathbf{G}_{e 3} & \mathbf{0} \\
\mathbf{0} & \mathbf{G}_{31} & \mathbf{G}_{3 e} & \mathbf{G}_{33} & \mathbf{R}^{T} \\
\mathbf{0} & \mathbf{0} & \mathbf{0} & \mathbf{R} & -\mathbf{M}_{2}
\end{array}\right]\left[\begin{array}{c}
\mathbf{A} \\
\mathbf{p}_{2 A} \\
\mathbf{p}_{2 e} \\
\mathbf{p}_{2 B} \\
\mathbf{B}
\end{array}\right]=\left[\begin{array}{c}
\tilde{\mathbf{M}}_{1} \\
\widetilde{\mathbf{Q}}^{T} \\
\mathbf{0} \\
\mathbf{0} \\
\mathbf{0}
\end{array}\right] .
$$

Equation (28) consists of $n_{T}\left(=m_{1}+n_{2}+m_{3}\right)$ simultaneous equations, which are solved to give the unknown modal amplitudes and pressures. The values for $\mathbf{B}$ may then be substituted into Eq. (9) in order to calculate the TL of the component section. Note that, provided the nodes in the finite element mesh are numbered correctly, Eq. (28) delivers a banded matrix that is also symmetrical (a result of the weighting function chosen when enforcing the pressure conditions). A further advantage of this MM approach is that one may choose $m_{1}<<n_{1}$ (and/or $m_{3}<<n_{3}$ ) so that the size of the problem is reduced, although for interior problems it is unlikely that this will significantly reduce the value for $n_{T}$, since normally $n_{2}>>n_{1}, n_{3}$.

\section{B. Point collocation}

In this section, matching conditions are enforced using the PC method described by Kirby and Lawrie $^{24}$. This involves matching over discrete points on $\Gamma_{A}$ and $\Gamma_{B}$. Here, the points chosen in 
adjacent regions must be identical, and so the number and location of the nodes generated in the eigensolution for the uniform duct sections must coincide with the location and number of the nodes generated in the finite element discretisation process for $\Gamma_{A}$ and $\Gamma_{B}$. This also requires that the number of modes used in the expansion of the sound pressure fields in regions $R_{1}$ and $R_{3}$ must also equal the number of collocation points on $\Gamma_{A}$ and $\Gamma_{B}$, respectively. Moreover, satisfying the velocity matching conditions at discrete points, rather than in the integral sense, means that the surface integrals in Eq. (8) are not carried out in the normal way; instead they are removed from Eq. (8) and enforced separately. Thus, after applying the boundary conditions listed in the MM approach, the velocity matching conditions may be written as

$$
\sum_{n=0}^{n_{1}} A_{n} \lambda_{n} \boldsymbol{\Phi}_{n}+\frac{i}{k_{1} \beta_{1}} \frac{\partial \mathbf{p}_{2 A}}{\partial x}=\sum_{n=0}^{n_{F}} F_{n} \lambda_{n} \boldsymbol{\Phi}_{n}
$$

and

$$
\sum_{j=0}^{n_{3}} B_{j} \gamma_{j} \boldsymbol{\Psi}_{j}-\frac{i}{k_{3} \beta_{3}} \frac{\partial \mathbf{p}_{2 B}}{\partial x}=\mathbf{0}
$$

Here, vectors $\boldsymbol{\Phi}$ and $\boldsymbol{\Psi}$ hold values of the duct eigenfunctions at nodal locations equivalent to those chosen for the finite element mesh in region $R_{2}$, but in regions $R_{1}$ and $R_{3}$, respectively. Similarly, the pressure matching conditions give

$$
-\sum_{n=0}^{n_{1}} A_{n} \boldsymbol{\Phi}_{n}+\mathbf{p}_{2 A}=\sum_{n=0}^{n_{F}} F_{n} \boldsymbol{\Phi}_{n}
$$

and

$$
-\sum_{j=0}^{n_{3}} B_{j} \boldsymbol{\Psi}_{j}+\mathbf{p}_{2 B}=\mathbf{0}
$$


A requirement of the collocation approach is that all of the eigensolutions obtained for regions $R_{l}$ and $R_{3}$ are used when enforcing Eqs. (29)-(32). These eigensolutions deliver a set of eigenmodes for which only about $20 \%$ are accurate; however, this does not cause any difficulties provided one uses a sufficient number of accurate eigenmodes to obtain a converged solution for the sound pressure field at each node. This issue is discussed in detail by Kirby and Lawrie ${ }^{24}$ but will also be reviewed here in the following section, in which PC predictions will be compared with MM predictions (that may use only "accurate" eigensolutions). The problem may finally be written as

$$
\left[\begin{array}{ccccc}
\lambda_{n} \boldsymbol{\Phi}_{n} & \frac{i}{k_{1} \beta_{1}} \frac{\partial \mathbf{N}}{\partial x} & \mathbf{0} & \mathbf{0} & \mathbf{0} \\
-\boldsymbol{\Phi}_{n} & \mathbf{G}_{11} & \mathbf{G}_{1 e} & \mathbf{G}_{13} & \mathbf{0} \\
\mathbf{0} & \mathbf{G}_{e 1} & \mathbf{G}_{e e} & \mathbf{G}_{e 3} & \mathbf{0} \\
\mathbf{0} & \mathbf{G}_{31} & \mathbf{G}_{3 e} & \mathbf{G}_{33} & -\boldsymbol{\Psi}_{j} \\
\mathbf{0} & \mathbf{0} & \mathbf{0} & -\frac{i}{k_{3} \beta_{3}} \frac{\partial \mathbf{N}}{\partial x} & \boldsymbol{\gamma}_{j}
\end{array}\right]\left[\begin{array}{c}
A_{n} \\
\mathbf{p}_{2 A} \\
\mathbf{p}_{2 e} \\
\mathbf{p}_{2 B} \\
B_{j}
\end{array}\right]=\left[\begin{array}{c}
\sum_{n=0}^{n_{F}} F_{n} \lambda_{n} \boldsymbol{\Phi}_{n} \\
\sum_{n=0}^{n_{F}} F_{n} \boldsymbol{\Phi}_{n} \\
\mathbf{0} \\
\mathbf{0} \\
\mathbf{0}
\end{array}\right] .
$$

Equation (33) consists of $n_{T}\left(=n_{1}+n_{2}+n_{3}\right)$ simultaneous equations, which are solved to give the unknown modal amplitudes and pressures. Note that Eq. (33) delivers a banded matrix, but this matrix is not symmetric. Note also that, when solving Eq. (33), it is necessary to multiply each of the terms that appear in Eqs. (31) and (32) by a scaling factor in order to enforce these equations in the final matrix (in the examples that follow $1 \times 10^{10}$ is used). 


\section{RESULTS AND DISCUSSION}

The methodology outlined in the previous section is validated here by comparison, where possible, with analytic predictions. Accordingly, this section begins by comparing predictions against analytic solutions for a simple expansion chamber and then proceeds to examine a converging-diverging duct. Finally, predictions are presented for a cylinder with a circular cross-section. In each case, a regular (circular or rectangular) geometry is chosen for regions $R_{l}$ and $R_{3}$, and a symmetric component section is also chosen. This allows for a reduction in the problem to two dimensions, which facilitates comparison with analytic theory and also results presented in the literature. In addition, where air is present, the speed of sound is taken to be $c_{0}=343.2244 \mathrm{~m} / \mathrm{s} ; k_{1}=k_{3}=\omega / c_{0}$, and $\beta_{1}=\beta_{3}=1$. For the finite element discretisation, a mesh consisting of either eight noded quadrilateral or six noded triangular isoparametric elements is used. Furthermore, it is normally accepted that at least seven to ten nodes per wavelength are required in order to achieve reasonable accuracy when using the FEM. Accordingly, for all the results that follow, at least seven nodes per wavelength have been used in the axial and radial directions.

\section{A. Expansion chamber}

The acoustic performance of an empty expansion chamber is well understood, and so provides a convenient example with which to begin validation of both methods. It is assumed here that the expansion chamber contains only air and has a circular cross-section of radius $r_{2}$; the inlet/outlet ducts are also assumed to be circular, both with radius $r_{l}$. After drawing a line of symmetry, the problem may be represented, as shown in Figs. 2(a) and 2(b). Here, two separate figures have 
been drawn in order to show that the position of planes A and B is arbitrary, provided that they pass through the inlet and outlet pipes. Clearly, one may extend region $R_{2}$ well into the inlet and outlet ducts, although this will be computationally more expensive since extra elements are required. In view of the arbitrary position of planes $\mathrm{A}$ and $\mathrm{B}$, predictions are presented here for different locations and the convergence of each model is investigated in order first to arrive at an optimum approach. Accordingly, a representative expansion chamber is chosen here, with $r_{1}=37 \mathrm{~cm}, r_{2}=76.2 \mathrm{~cm}$ and $L=315 \mathrm{~cm}$. In Fig. 3, TL predictions are presented for the MM (with $m_{1}=n_{1}$, and $m_{3}=n_{3}$ ) and PC methods, with and without mesh extensions. Here, the mesh extensions in the inlet and outlet ducts are assumed to be one element deep (in the $x$ direction), but may contain more than one element in the radial direction, see Fig. 2(b). Note that, for a simple area discontinuity, a modal expansion of the sound pressure field away from the discontinuity must deliver the same solution as that obtained using additional finite elements. Accordingly, there is nothing to be gained from adding additional elements to the mesh extensions, at least for the simple area discontinuities such as those found in this example.

In Fig. 3, TL predictions using MM and PC are shown at frequencies of 300 and $3240 \mathrm{~Hz}$. Here, problems with convergence are clearly evident in the PC approach, but only when no mesh extensions are in place (note that the MM solutions with and without extensions are identical to at least 10 decimal places). The discrepancy between the two methods is likely to be caused by difficulties in enforcing the matching conditions at individual nodes over $\Gamma_{A}$ and $\Gamma_{B}$ when no extensions are present in the collocation method. At this corner, acoustic scattering will be pronounced and it is likely that a relatively large number of evanescent modes will be required when using a Fourier series to represent the pressure and velocity fields in $R_{1}$ and $R_{3}$. This is 
likely to cause relatively slow rates of convergence, as seen for the PC method in Fig. 3 when no mesh extensions are present. This trend has also been observed at other frequencies and for other chamber geometries (not shown here). In contrast, a more convergent system of equations is apparent when using mesh extensions since those evanescent modes at the silencer edge do not influence the sound pressure field away from the edge. It is noticeable, however, that if one uses average values by integrating over the cross-section, these problems disappear and the MM approach is seen to converge very quickly, even when no mesh extensions are present. Clearly, the MM approach is the more convergent of the two methods, at least in terms of computing component TL.

In view of the improved efficiency of the MM method all future results reported here will be obtained using this method. It is of interest then to examine the potential savings that may be realised by setting $m_{1}<n_{1}$, and $m_{3}<n_{3}$. In Table I, TL values obtained using the MM method are compared for the expansion chamber at frequencies of 300 and $3240 \mathrm{~Hz}$. Here, the value of $m_{1}\left(=m_{3}\right)$ is systematically increased up to $m_{1}=n_{1}=n_{3}$ for a relatively dense finite element mesh $\left(n_{2}=2735\right)$. It is evident in Table I that at low frequencies convergence is very fast and one needs only to include one or perhaps two modes in the calculations. It is not surprising, however, that as the frequency is increased more modes are necessary and at $3240 \mathrm{~Hz}$ at least seven modes are required in order to achieve comparable levels of accuracy. Therefore, when using this hybrid approach it would appear to be sensible, at least at higher frequencies, to properly examine solution convergence before reducing values of $m_{1}$ and $m_{3}$. Alternatively, given that $m_{1}$ is normally a lot less than $n_{2}$, the potential savings from setting $m_{1}<n_{1}$ are likely to be small, and so it is convenient simply to set $m_{1}=n_{1}$ and $m_{3}=n_{3}$ when solving the problem. 
In view of this, all the TL computations that follow are computed with $m_{1}=n_{1}$ and $m_{3}=n_{3}$, and convergence is examined by modifying $n_{2}$.

Transmission loss predictions for the expansion chamber are presented in Fig. 4. These predictions are compared with a plane wave analytic solution and an analytic MM solution that includes higher order modes. For purposes of comparison, the TL is plotted against a modified Helmholtz number $k_{c}$, where $k_{c}=2 \pi r_{1} f / 3.8317 c_{0}$, so that a value of $k_{c}=1$ represents the value at which higher order modes are "cut-on" in regions $R_{1}$ and $R_{3}$. For this expansion chamber, $k_{c}=1$ equates to a frequency of $5657 \mathrm{~Hz}$ and a wavelength of $\lambda=1.64 r_{1} \mathrm{~m}$. In Fig. 4 the hybrid method overlays the analytic MM solution over virtually the entire frequency range, and very good agreement with the plane wave model is also observed at lower frequencies, as one would expect. Moreover, the results plotted here have been obtained with a relatively modest number of degrees of freedom, especially at lower frequencies $\left(n_{T}=75\right.$ up to $k_{c}=0.1$, rising to $n_{T}=1127$ at $k_{c}=1$ ). Of course, one would not consider using this technique to design expansion chambers, as analytic techniques would be much faster; however, the results for the expansion chamber clearly demonstrate the efficacy of the hybrid method for interior problems and this will now be investigated further by examining non-uniform geometries.

\section{B. Converging-Diverging duct}

A simple non-uniform geometry that is often found in the ductwork is the converging-diverging duct. For example, Selamet and Easwaran ${ }^{2}$ studied the so-called Herschel-Venturi tube, which has a circular cross-section and is used in flow measurements; Lau and Tang $^{9}$ studied $^{2}$ constrictions that are often found in a rectangular ventilation duct. To accommodate both 
rectangular and circular geometries a representative convergent-divergent duct geometry is shown in Fig. 5. This assumes that a line of symmetry may be drawn through both geometries, remembering that for the circular geometry the solution must be integrated over the circular cross-section in the normal way. A well-known analytic solution exists for the circular HerschelVenturi tube ${ }^{2}$, which permits direct comparison with the current method. Accordingly, in Fig. 6 the hybrid method is compared with analytic predictions for values of the modified Helmholtz number $k_{c}$ up to unity. Here, two of the geometries studied by Selamet and Easwaran ${ }^{2} \operatorname{are}^{2}$ examined, and for each example $2 r_{1}=4.859 \mathrm{~cm}$ and $L=r_{2}$; the other dimensions are (a) $r_{2}=0.5 r_{1}, \quad L_{c}=6.554 \mathrm{~cm}$, and $L_{d}=18.534 \mathrm{~cm}$ and (b) $r_{2}=0.25 r_{1}, \quad L_{c}=9.831 \mathrm{~cm}$, and $L_{d}=27.8 \mathrm{~cm} . \quad$ In addition, when $k_{c} \leq 0.2, \quad n_{T}=243 ; 0.2<k_{c} \leq 0.5, \quad n_{T}=655 ;$ and $0.5<k_{c} \leq 1, n_{T}=1021$. Here, $k_{c}=1$ equates to an upper frequency limit of $8614 \mathrm{~Hz}$, and $\lambda=1.64 r_{1} \mathrm{~m}$. Good agreement between the numerical and analytic solutions is observed in Fig. 6 for values up to $k_{c}=1$, further validating the approach.

Lau and Tang $^{9}$ examined a converging-diverging restriction in a rectangular ventilation duct, which is much larger than the Herschel-Venturi tube studied by Selamet and Easwaran ${ }^{2}$. Accordingly, to cover a relevant frequency range, say up to $1 \mathrm{kHz}$, it is necessary to go to a much higher modified Helmholtz number and this requires the inclusion of higher order modes in the inlet and outlet ducts. A significant advantage of the hybrid method is that specifying a nonreflecting boundary is trivial when higher order modes are present, and it is also straightforward to quantify the true sound power propagating in region $R_{3}$. In Fig. 7, TL predictions for three of the rectangular convergent-divergent sections studied by Lau and Tang are plotted against a 
modified Helmholtz number, $k_{r}=2 r_{1} f / c_{0}$ (so that higher order modes in $R_{3}$ cut on at $\left.n k_{r}, \mathrm{n}=1,2, . . \infty\right)$. Here, when $k_{r} \leq 1, n_{T}=179 ; 1<k_{r} \leq 2, n_{T}=429 ;$ and $2<k_{r} \leq 3, n_{T}=667$ Also, a value of $k_{r}=3$ equates to an upper frequency limit of $1030 \mathrm{~Hz}$ and $\lambda=0.67 r_{1} \mathrm{~m}$. Note that $r_{1}$ now represents the half width of the rectangular section in regions $R_{1}$ and $R_{3}$, and that only those modes symmetric about the centre line of the duct are considered in the analysis. The TL values presented in Fig. 7 generally compare well with Lau and Tang's predictions ${ }^{9}$ up to $k_{r}=1$, although for case (c) some discrepancies are evident as $k_{r} \rightarrow 1$. TL values for $k_{r}>1$ are, however, very different from those reported by Lau and Tang and it is noticeable that negative values no longer appear. This is because the hybrid approach includes all modes in the TL calculations, rather than examining individual modes. The TL predictions in Fig. 7 assume that plane wave propagation is present in the inlet duct; however, for $k_{r}>1$ multi-mode sound propagation is also possible in the inlet duct, especially if the noise source is a fan. Thus, in Fig. $8 \mathrm{TL}$ predictions are presented for an incident sound field containing EMED, ${ }^{24}$ with the same value for $n_{T}$ as used in Fig. 7. It is evident in Fig. 8 that the TL of the converging-diverging section changes significantly if the incident sound field contains higher order modes. For these examples, the TL appears to depend strongly on the respective geometries and frequency of excitation and so no characteristic trends are evident. These results do show, however, that a system is likely to behave very differently if driven by a sound source that contains higher order modes.

In addition to predicting TL, Lau and Tang ${ }^{9}$ also plotted the sound pressure distribution. This provides a further opportunity for validating the hybrid method and so in Fig. 9 three different plots of absolute sound pressure level are presented, which have been chosen to match the 
Helmholtz number and contraction geometries presented in Fig. 6 of Ref. 9. A careful comparison with Lau and Tang's results indicates generally good agreement, although it is noticeable that for $k_{r}=1$ a more resonant response is observed in Fig. 9. Here, it is possible that any discrepancies between the two sets of results are caused by reflections from Lau and Tang's downstream boundary contaminating the pressure field when higher order modes are present.

\section{Cylinder}

A classical problem in duct acoustics is sound propagation over a cylinder placed on the centreline of a rectangular duct, see for example Duan et al. ${ }^{7}$ A circular cylinder is examined here, which has the geometry shown in Fig. 10. The MM analysis carried out in the previous two sections sought to minimise, as far as possible, the number of degrees of freedom required and so planes A and B were located at duct discontinuities. However, when more complicated geometries are present, it is important to ensure that significant distortion within the finite elements used to discretise region $R_{2}$ is avoided in order to minimise numerical errors. One must, therefore, be careful when locating planes A and B, and here these planes are moved a distance $L_{e}$ from the surface of the cylinder in order to minimise element distortion close to the cylinder. The convergence of the TL is then investigated in the same way as before, noting that it is essential to use eight noded quadrilateral and/or six noded triangular isoparametric elements in order to accurately reproduce the geometry of a semi-circle. The convergence of the TL for $r_{2}=0.8 r_{1}$ is reviewed in Table II for different values of $L_{e}$. Here, the effect of moving planes A and B away from the cylinder can be seen in the rate at which the solution converges, although if the value of $L_{e}$ is relatively small then numerical errors appear to inhibit convergence. From Table II, a value of $L_{e}=0.5 r_{2}$ is generally seen to provide an optimum balance between 
competing requirements and it is this value for $L_{e}$ that is used in the TL calculations that follow. It should be noted, however, that Table II represents a worst case scenario for those cylinders studied here; at lower frequencies (and for plane wave excitation) convergence is normally much faster and there is little difference between the TL values when $L_{e}$ is altered. Nevertheless, it is good practice here to carefully investigate convergence for all complex geometries, noting that the rate of convergence will depend on the length of the mesh extension, the geometry of the mesh chosen, and the frequency of excitation. After establishing convergence over a range of frequencies, the TL for a circular cylinder with $r_{2}=0.4 r_{1}, 0.6 r_{1}$, and $0.8 r_{1}$ is shown in Fig. 11 for multi-mode (EMED) forcing, with $n_{T}=79$ for $k_{r} \leq 1 ; n_{T}=251$ for $1<k_{r} \leq 2$; and $n_{T}=687$ for $2<k_{r} \leq 3$. Here, a value of $k_{r}=3$ equates to an upper frequency limit of $1030 \mathrm{~Hz}$ and $\lambda=0.67 r_{1} \mathrm{~m}$. In Fig. 11 it is evident that for $k_{r}<1$ the TL of the cylinder is relatively small, even when $80 \%$ of the duct is blocked, although the TL still exhibits the dome like behaviour seen for the converging-diverging ducts. However, when higher order modes propagate, the TL is seen to increase significantly and those modes that cut on at $k_{r}=2$ and $k_{r}=3$ strongly influence the sound TL.

In the previous two sections, the TL predictions were partially validated by comparing the hybrid method against analytic predictions. An alternative method for validating the predictions is to examine the relative error in the energy balance over the cylinder. Therefore, if the normalised reflected and transmitted sound power are denoted by $W_{\text {Ref }}$ and $W_{\text {Trans }}$, respectively, then the percentage error $\Delta E$ in the sound power is given by $\Delta E=100 \times\left|1-W_{\text {Ref }}-W_{\text {Trans }}\right|$, provided that $W_{\text {Ref }}$ and $W_{\text {Trans }}$ are normalised against the incident sound power. Values for $\Delta E$ may then be 
computed over the entire frequency range for each cylinder. Here, for $k_{r}<1, \Delta E<10^{-12} \%$; for $1 \leq k_{r}<2, \Delta E<0.001 \%$; and for $2 \leq k_{r}<3, \Delta E<0.03 \%$. These values are representative of each cylinder studied and also apply (and in most cases are much lower for $k_{r}>1$ ) to the expansion chamber and converging diverging ducts studied previously. Accordingly, the values quoted for $\Delta E$ are sufficiently small to provide confidence in the accuracy of the hybrid method and in the results presented here.

\section{CONCLUSIONS}

A hybrid numerical method that combines a wave based modal solution with a standard finite element solution has been reviewed here. The main advantage of this method is that it avoids the need to mesh long uniform sections of ductwork, but at the same time retains the flexibility of the FEM so that relatively short but geometrically complex component sections may be modelled accurately. Two different approaches in enforcing the continuity conditions over the interface between the uniform and non-uniform sections of the ductwork are analysed: MM and PC. It is observed that the TL predictions obtained using the MM method converge faster than those obtained using PC. Moreover, to ensure good rates of convergence, the PC method requires continuity conditions to be enforced away from duct discontinuities in order to avoid corner nodes. Both the PC and MM methods retain a banded matrix, although only the MM technique will deliver a symmetrical matrix (provided one chooses an appropriate weighting function when enforcing continuity of pressure). Accordingly, the MM version of the hybrid method is capable of retaining all of the benefits of the traditional FEM. Furthermore, the hybrid method delivers a 
very straightforward way of calculating the component TL, which includes a computationally efficient specification of a non-reflecting boundary condition as well as accommodating higher order modes in the inlet and outlet ducts. This then allows sound propagation to be analysed at frequencies above the cut-on frequency of the first higher order mode in the outlet duct as well as allowing the introduction of a multi-modal incident sound field.

The hybrid method is validated here by comparing numerical predictions against analytic solutions, as well as other results reported in the literature, and good agreement is observed in each case. In order to facilitate the validation of the method, the examples chosen here have been deliberately restricted to relatively simple two-dimensional geometries and so this remains to be demonstrated for more complex three-dimensional component geometries that include inlet/outlet ducts of arbitrary cross-section. Of course, for fully three-dimensional models one may expect the number of degrees of freedom required to achieve a converged solution to grow rapidly, but this is true for any numerical model and for most fully three-dimensional shapes one is left with little alternative. The results presented here have been restricted to one component section only, although it is relatively straightforward to add further components since the modal solution in the outlet duct may readily be used as the incident sound pressure field for another component section further downstream. In this way one can build up a number of (multi-mode) transfer matrices and so examine the interaction between different components in, say, a ventilation system without worrying about the distance between each component. Thus, the hybrid method has the potential to provide an efficient method with which to study sound propagation over complex non-uniform components in a duct and also to study multiple components in order to build up a picture of how sound interacts throughout a ducting system. 


\section{REFERENCES}

1. J.H. Miles, "Acoustic transmission matrix of a variable area duct or nozzle carrying a compressible subsonic flow,” J. Acoust. Soc. Am. 69, 1577-1586 (1981).

2. A. Selamet and V. Easwaran, "Wave propagation and attenuation in Herschel-Venturi tubes," J. Acoust. Soc. Am. 101, 936-942 (1997).

3. A. Boström, "Transmission and reflection of acoustic waves by an obstacle in a waveguide," Wave Motion 2, 167-184 (1980).

4. D.V. Evans, M. Levitin and D. Vassiliev, "Existence theorems for trapped modes," J. Fluid Mech. 261, 21-31 (1994).

5. C.M. Linton and M. McIver, "Trapped modes in cylindrical waveguides," Q. J1 Mech. appl. Math. 51, 389-412 (1998).

6. C.M. Linton, M. McIver, P. McIver, K. Ratcliffe and J. Zhang, "Trapped modes for offcentre structures in guides," Wave Motion 36, 67-85 (2002).

7. Y. Duan, W. Koch, C.M. Linton and M. McIver, "Complex resonances and trapped modes in ducted domains," J. Fluid Mech. 571, 119-147 (2007).

8. S.K. Tang and C.K. Lau, "Sound transmission across a smooth nonuniform section in an infinitely long duct," J. Acoust. Soc. Am. 112, 2602-2611 (2002).

9. C.K. Lau and S.K. Tang, "Sound transmission across duct constrictions with and without tapered sections," J. Acoust. Soc. Am. 117, 3679-3685 (2005).

10. J-H. Jeong, J-G. Ih and B-C. Lee, "A guideline for using the multi-domain BEM for analysing the interior acoustic field," J. Comput. Acoust. 3, 403-424 (2003). 
11. S. Bilawchuk and K.R. Fyfe, "Comparison and implementation of the various numerical methods used for calculating transmission loss in silencer systems," Appl. Acoust. 64, 903916 (2003).

12. O.Z. Mehdizadeh and M. Paraschivoiu, "A three-dimensional finite element approach for predicting the transmission loss in mufflers and silencers with no mean flow," Appl. Acoust. 66, 902-918 (2005).

13. D. Givoli, "High-order local non-reflecting boundary conditions: a review," Wave Motion 39, 319-326 (2004).

14. A. Mal and Z. Chang, "A semi-numerical method for elastic wave scattering calculations," Geophys. J. Int. 143, 328-334 (2000).

15. S.W. Liu, S. K. Datta and T.H. Ju, "Transient scattering of Rayleigh-Lamb waves by a surface-breaking crack: comparison of numerical simulation and experiment," J. Nondestruct. Eval. 10, 111-126 (1991).

16. G.C. Chinn, L.W. Epp and D.J. Hoppe, “A hybrid finite element method for axisymmetric waveguide-fed horns," IEEE Trans. Antennas Propagat. 44, 280-285 (1996).

17. H. Esteban, S. Cogollos, V.E. Boria, A. San Blas and M. Ferrando, "A new hybrid modematching/numerical method for the analysis of arbitrarily shaped inductive obstacles and discontinuities in rectangular waveguides," IEEE Trans. Microwave Theory Tech, 50, 12191224 (2002).

18. A. Monorchio, G. Manara, P. Grassi and D. Arena, "Analysis of waveguide components and inductive frequency-selective surfaces by using a hybrid mode matching-finite element technique with edge elements," Electromagnetics 24, 49-67 (2004). 
19. Y. Kagawa, T. Yamabuchi, T. Yoshikawa, S. Ooie, N. Kyouno and T. Shindou, "Finite element approach to acoustic transmission-radiation systems and application to horn and silencer design," J. Sound Vib. 69, 207-228 (1980).

20. R.J. Astley and A. Cummings, "A finite element scheme for acoustic transmission through the walls of rectangular ducts: comparison with experiment," J. Sound Vib. 92, 387-409 (1984).

21. M.G. Imhof, "Scattering of acoustic and elastic waves using hybrid multipole expansions finite element technique," J. Acoust. Soc. Am. 100, 1325-1338 (1996).

22. R.J. Astley, "FE mode-matching schemes for the exterior Helmholtz problem and their relationship to the FE-DtN approach," Commun. Numer. Methods Eng. 12, 257-267, (1996).

23. R.J. Astley and W. Eversman, "Acoustic transmission in non-uniform ducts with mean flow, part II: the finite element method,” J. Sound Vib. 74, 103-121 (1981).

24. R. Kirby and J.B. Lawrie, "A point collocation approach to modelling large dissipative silencers," J. Sound Vib. 286, 313-339 (2005).

25. R.J. Astley and A. Cummings, "A finite element scheme for attenuation in ducts lined with porous material: comparison with experiment," J. Sound Vib. 116, 239-263 (1987). 
Kirby, JASA

\begin{tabular}{|c|c|c|}
\hline \multicolumn{3}{|c|}{$\begin{array}{c}\text { Table I. Convergence of mode matching approach for } \\
\text { expansion chamber with plane wave forcing. }\end{array}$} \\
\hline & $T L(\mathrm{~dB})$ & $T L(\mathrm{~dB})$ \\
\hline$m_{1}\left(=m_{3}\right)$ & $\mathrm{F}=300 \mathrm{~Hz}$ & $\mathrm{~F}=3240 \mathrm{~Hz}$ \\
\hline 1 & 6.983130 & 5.859270 \\
\hline 2 & 6.978823 & 7.057272 \\
\hline 3 & 6.977517 & 7.237816 \\
\hline 4 & 6.976890 & 7.299525 \\
\hline 5 & 6.976516 & 7.328743 \\
\hline 6 & 6.976292 & 7.343553 \\
\hline 7 & 6.976051 & 7.357351 \\
\hline 8 & 6.975850 & 7.368384 \\
\hline 9 & 6.975679 & 7.377397 \\
\hline 10 & 6.975526 & 7.385279 \\
\hline 11 & 6.975378 & 7.392681 \\
\hline 12 & 6.975201 & 7.401415 \\
\hline$n_{1}=13$ & 6.975171 & 7.402905 \\
\hline
\end{tabular}


Kirby, JASA

\begin{tabular}{|c|c|c|c|c|}
\hline \multicolumn{5}{|c|}{$\begin{array}{c}\text { Table II. } \\
\text { Convergence of mode matching approach } \\
\text { for cylinder with EMED forcing. }\end{array}$} \\
\hline & $T L(\mathrm{~dB})$ at $\mathrm{F}=1000 \mathrm{~Hz}\left(k_{r}=2.914\right)$ for $r_{2}=0.8 r_{1}$ \\
\hline$n_{T}$ & $L_{e}=r_{2}$ & $L_{e}=0.5 r_{2}$ & $L_{e}=0.25 r_{2}$ & $L_{e}=0.1 r_{2}$ \\
\hline 53 & 24.0202 & 25.3502 & 29.3648 & 30.6231 \\
\hline 79 & 21.8790 & 29.1131 & 36.8324 & 33.7745 \\
\hline 115 & 16.1962 & 21.4286 & 24.2204 & 27.3093 \\
\hline 151 & 16.3297 & 21.6613 & 22.2208 & 23.1645 \\
\hline 251 & 19.4340 & 21.8583 & 22.4775 & 23.2662 \\
\hline 345 & 20.4249 & 21.3426 & 21.5664 & 21.7407 \\
\hline 519 & 20.8249 & 21.3660 & 21.5725 & 21.7412 \\
\hline 687 & 21.0402 & 21.2821 & 21.3552 & 21.4128 \\
\hline 855 & 21.1031 & 21.2574 & 21.2896 & 21.3145 \\
\hline 1099 & 21.1654 & 21.2598 & 21.2906 & 21.3146 \\
\hline 1607 & 21.2065 & 21.2511 & 21.2663 & 21.2780 \\
\hline 2523 & 21.2290 & 21.2454 & 21.2503 & 21.2541 \\
\hline
\end{tabular}


Kirby, JASA

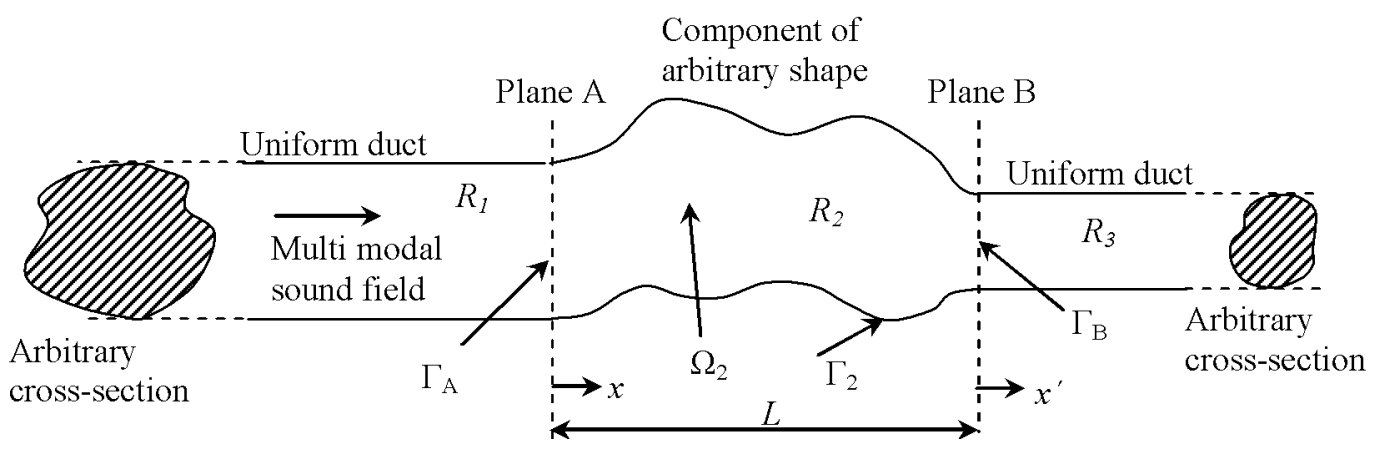

Figure 1. Geometry of duct. 
Kirby, JASA

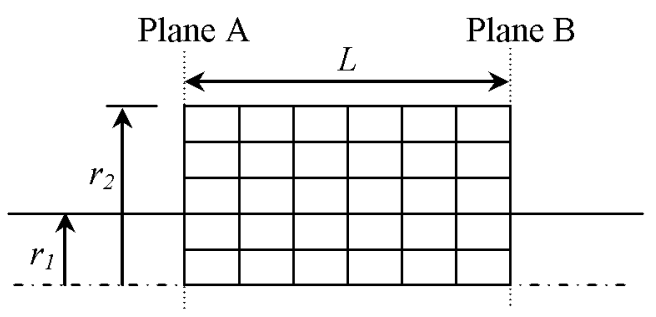

(a)

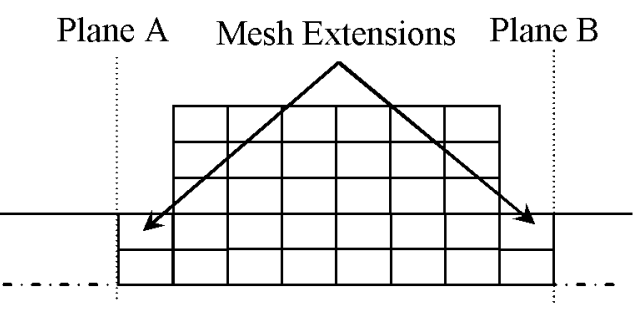

(b)

Figure 2. (a) Mesh for expansion chamber; (b) Extended mesh for expansion chamber. 
Kirby, JASA

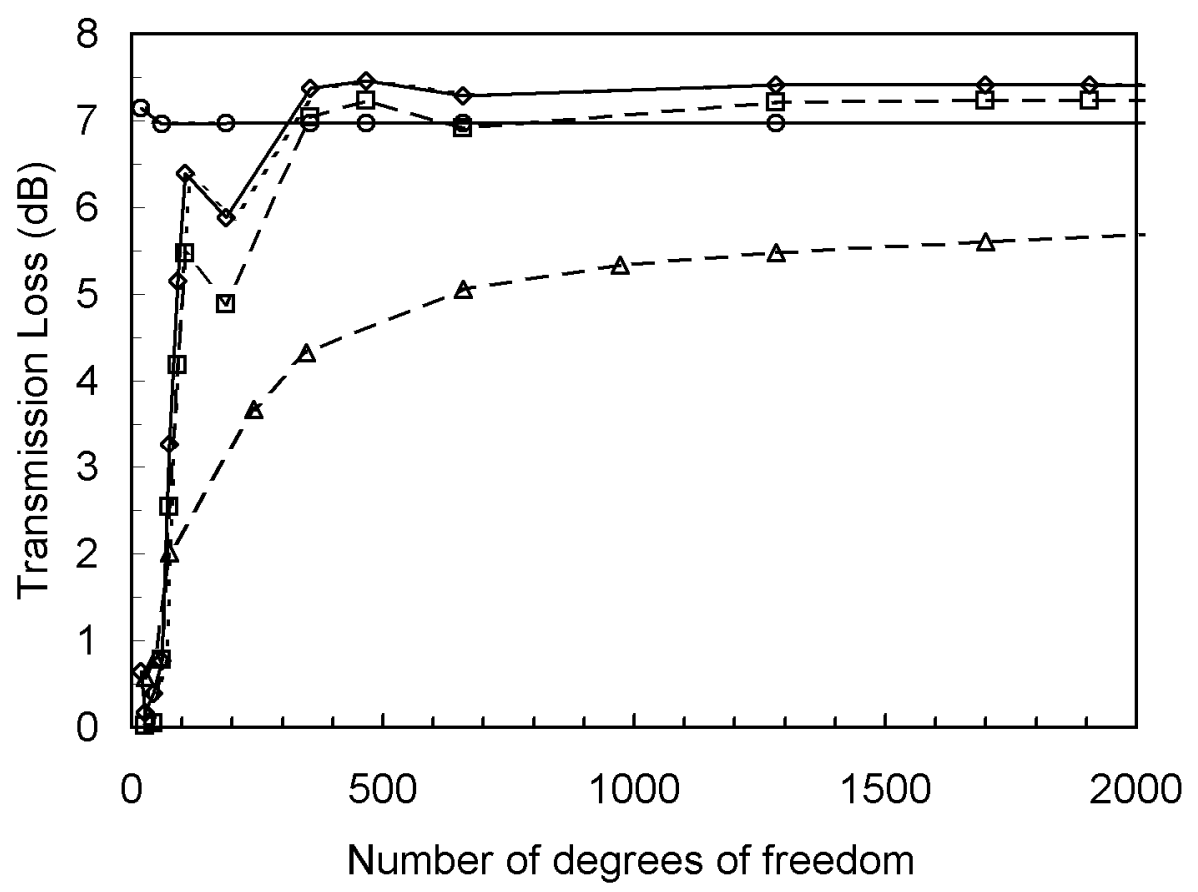

Figure 3. Convergence of TL for expansion chamber: $-\mathrm{O}-\mathrm{O}-$, MM, $300 \mathrm{~Hz}$; $\multimap-\diamond$, MM, $3240 \mathrm{~Hz} ;-\Delta-\Delta-$, PC no mesh extension, 300Hz; — - — —, PC no mesh extension, 3240Hz; - - - - - , PC mesh extensions (overlays MM for $300 \mathrm{~Hz}$ and at higher values of $n_{\mathrm{T}}$ for $\left.3240 \mathrm{~Hz}\right)$. 
Kirby, JASA

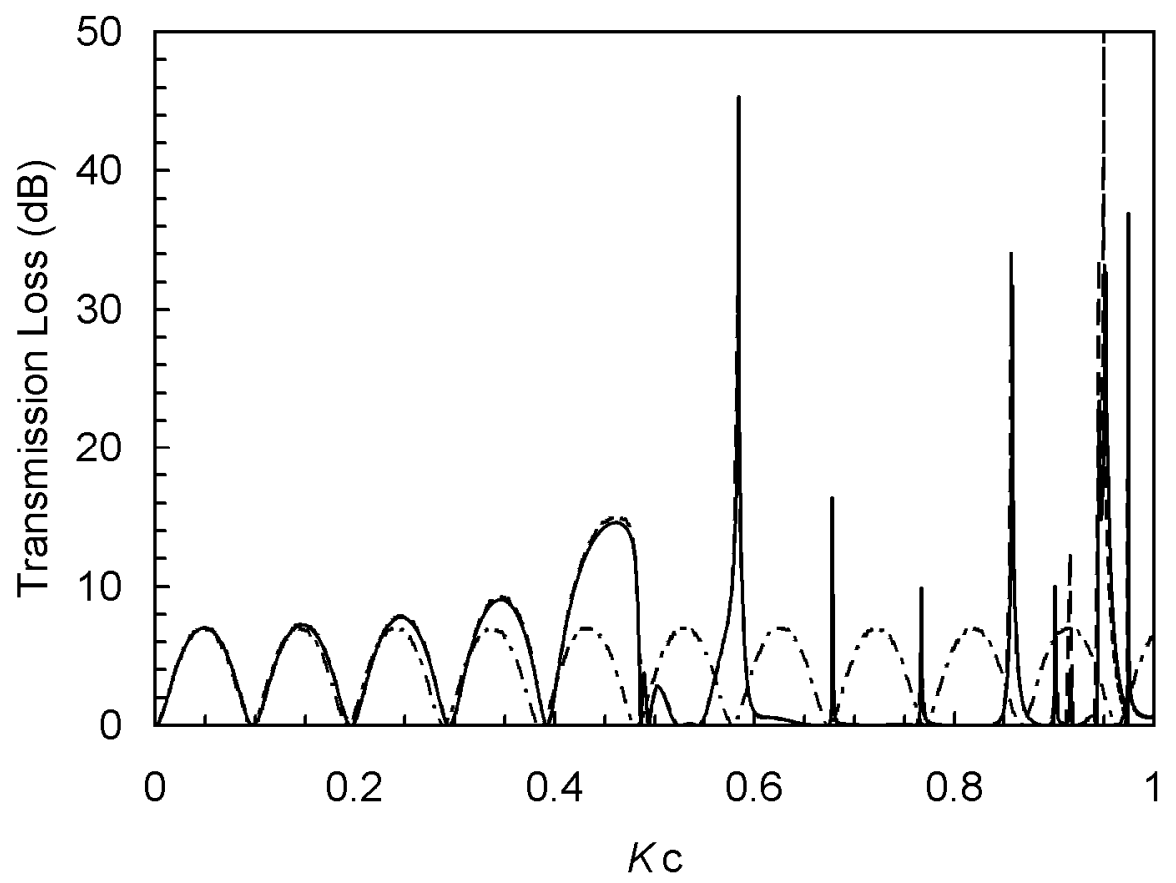

Figure 4. TL predictions for expansion chamber: - , hybrid method (MM); $--\frac{}{-}$ analytic mode matching (overlays hybrid method); $-\cdots--\ldots$, plane wave. 


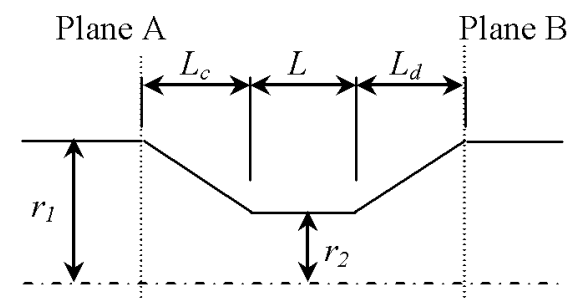

Figure 5. Geometry of converging-diverging duct. 
Kirby, JASA

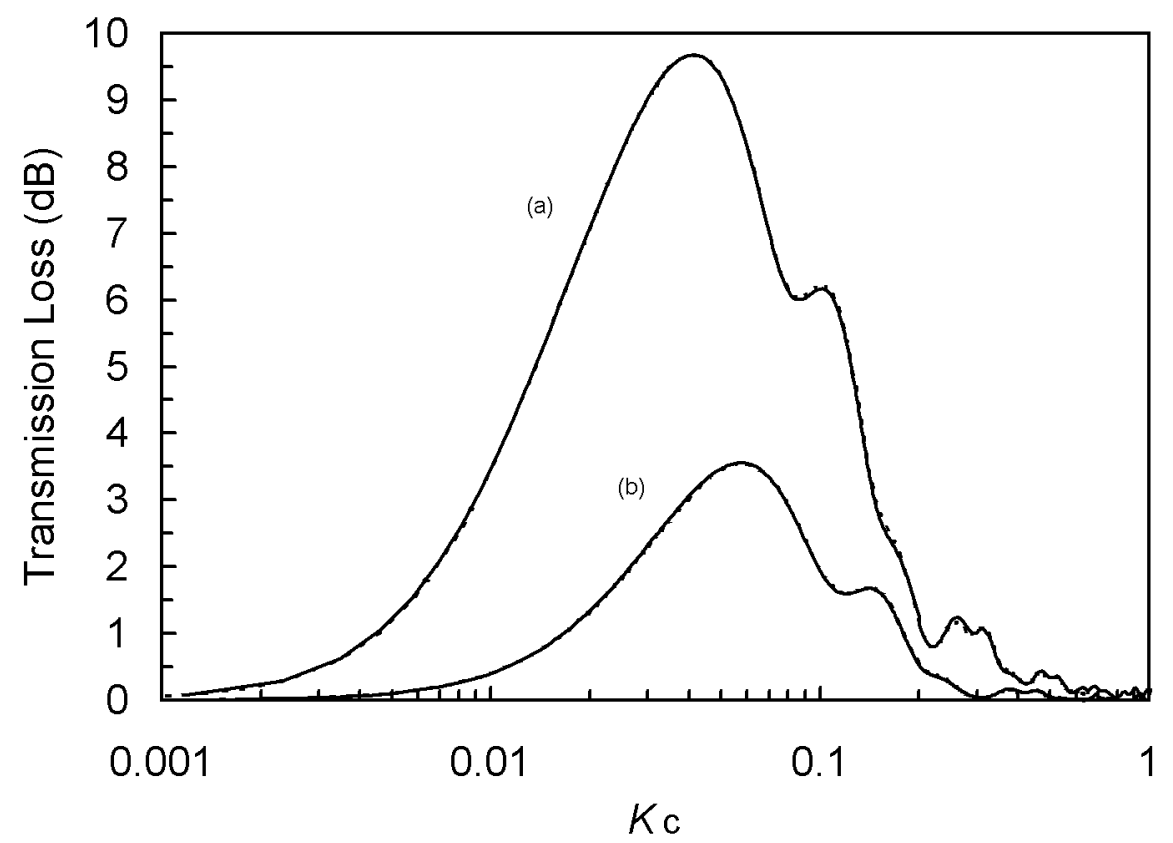

Figure 6. TL predictions for Selamet and Easwaran's converging-diverging duct $^{2}$ : hybrid method (MM); - - - - -, analytic method ${ }^{2}$ (overlays hybrid method). (a) $r_{2}=0.5 r_{1}$, $L_{c}=6.554 \mathrm{~cm}, L_{d}=18.534 \mathrm{~cm}$; (b) $r_{2}=0.25 r_{1}, L_{c}=9.831 \mathrm{~cm}, L_{d}=27.8 \mathrm{~cm}$. 
Kirby, JASA

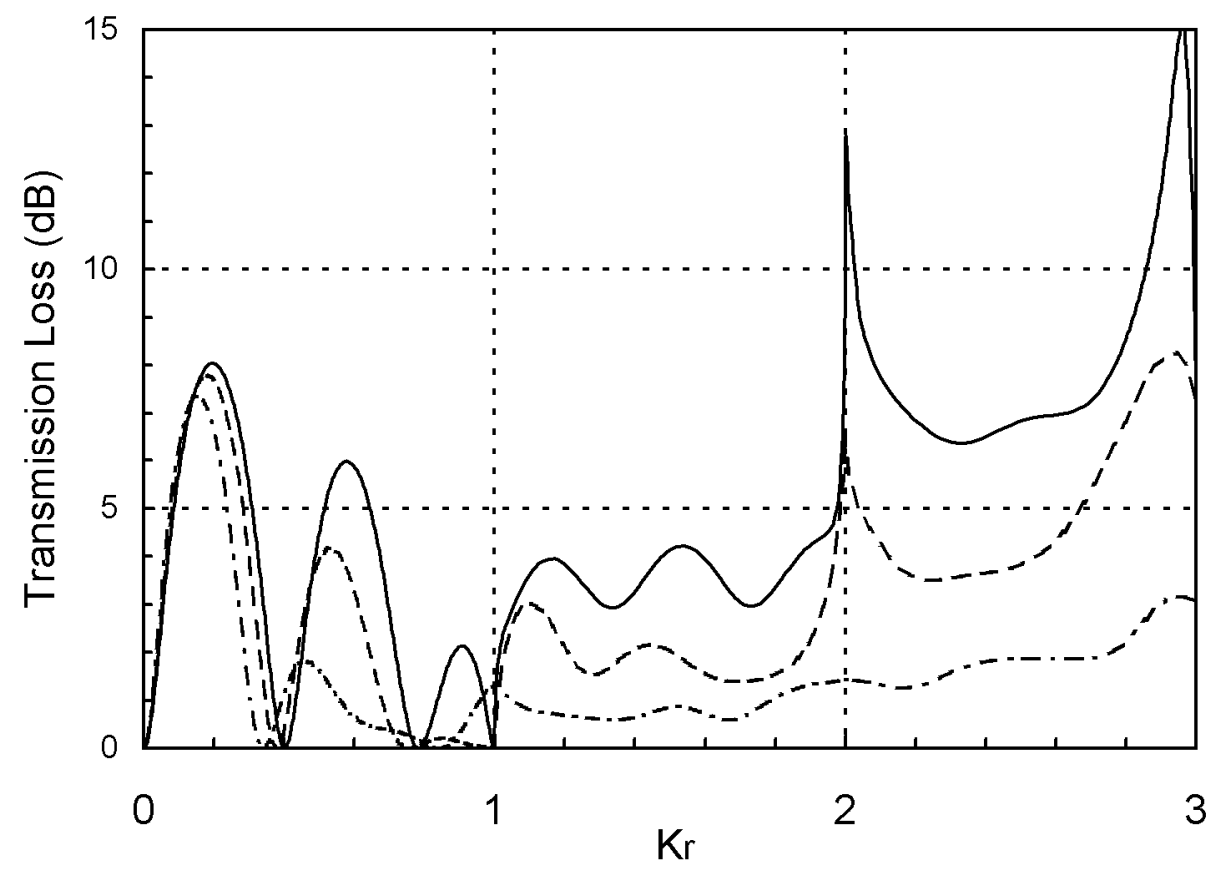

Figure 7. Hybrid method (MM) predictions for Lau and Tang's converging-diverging duct ${ }^{9}$, with plane wave excitation. For each plot, $r_{1}=0.5 \mathrm{~m}, r_{2}=0.2 r_{1}$, and $L=2 r_{1}$. (a) $\longrightarrow$, $L_{c}=L_{d}=0.2309 \mathrm{~m} ;(\mathrm{b}) ;-\longrightarrow-, L_{c}=L_{d}=0.4 \mathrm{~m} ;(\mathrm{c})--\longrightarrow-L_{c}=L_{d}=0.6928 \mathrm{~m}$. 
Kirby, JASA

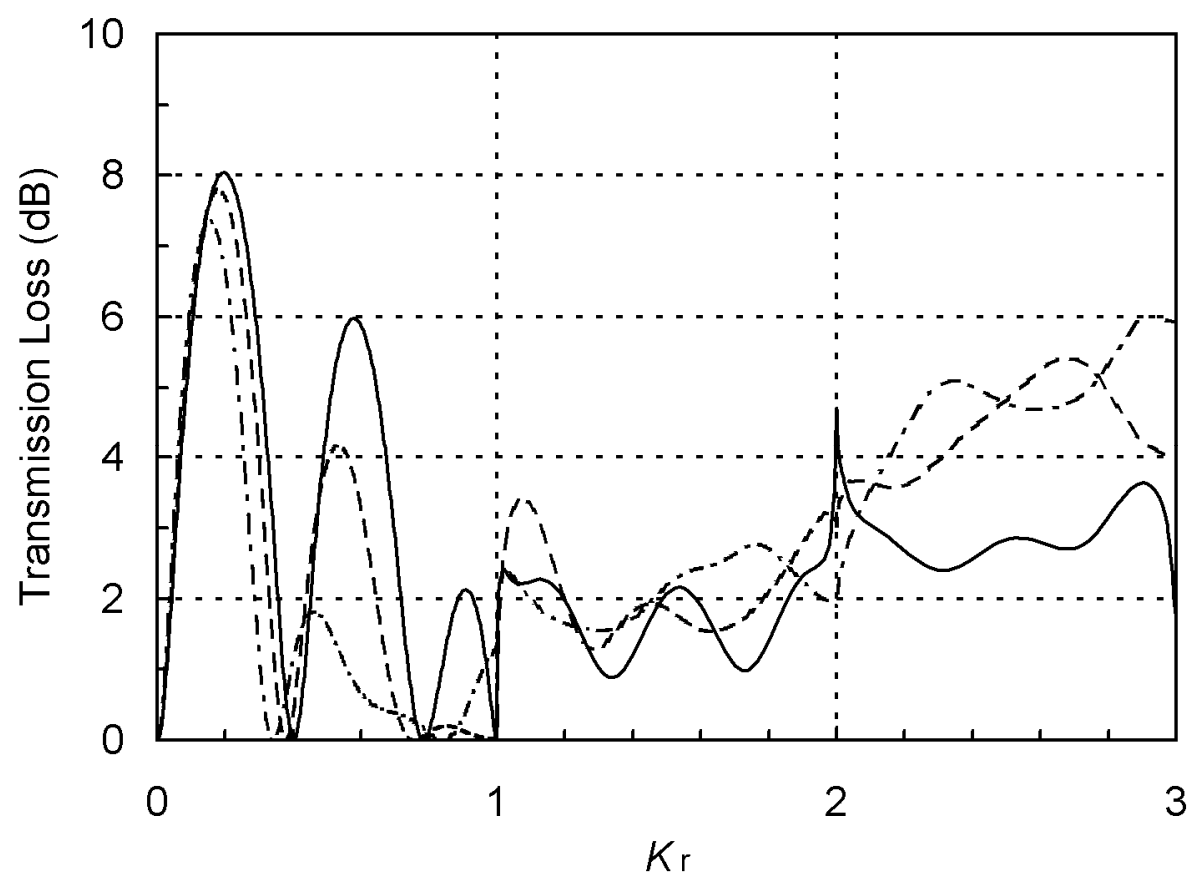

Figure 8. Hybrid method (MM) predictions for Lau and Tang's converging-diverging duct ${ }^{9}$, with multi-mode (EMED) excitation. For each plot, $r_{1}=0.5 \mathrm{~m}, r_{2}=0.2 r_{1}$, and $L=2 r_{1}$. (a)

$\longrightarrow, L_{c}=L_{d}=0.2309 \mathrm{~m} ;(\mathrm{b}) ;-\quad-\quad L_{c}=L_{d}=0.4 \mathrm{~m} ;$ (c) $-\cdots--$ $L_{c}=L_{d}=0.6928 \mathrm{~m}$. 
Kirby, JASA
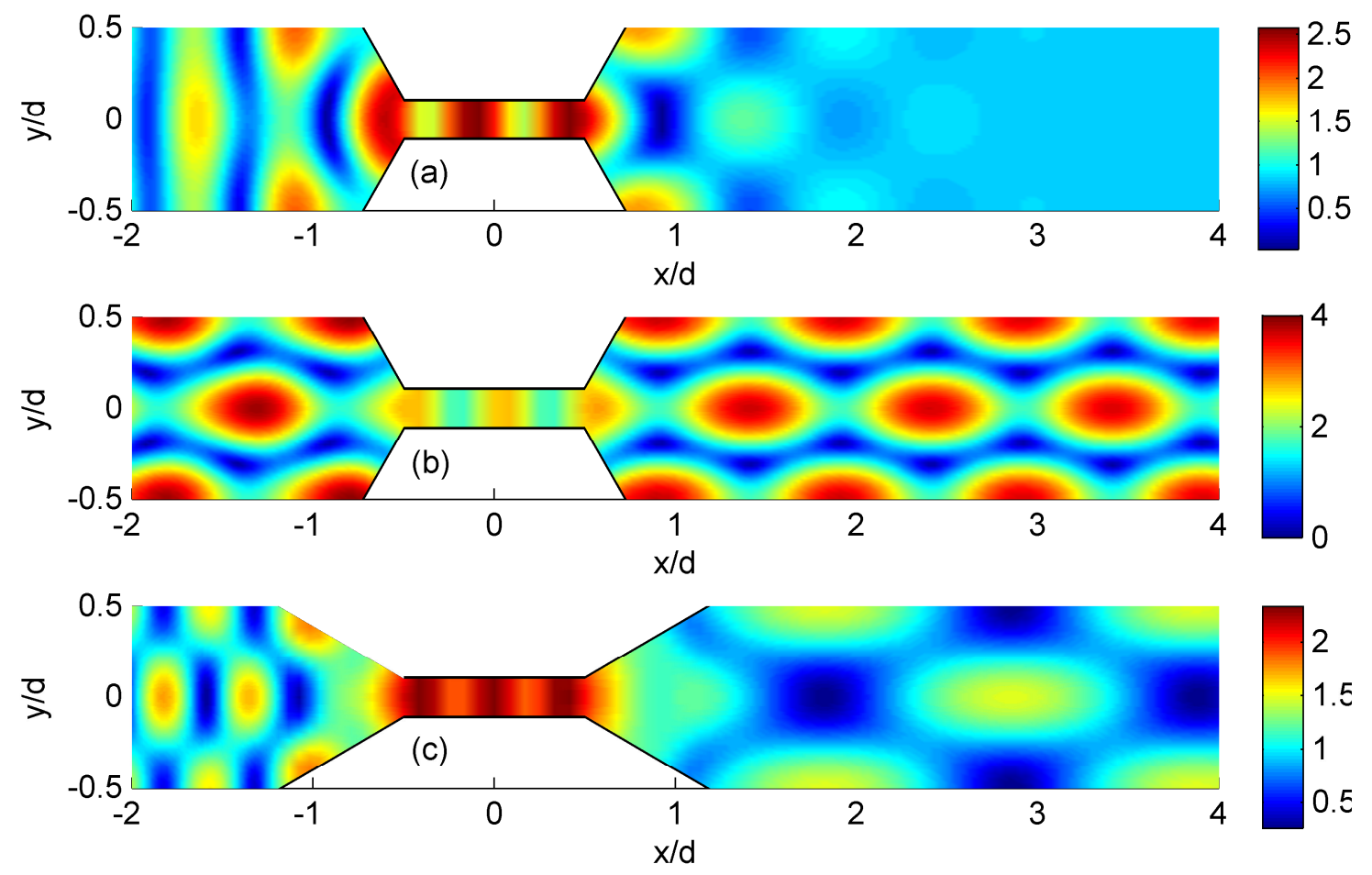

Figure 9. Magnitude of sound pressure for Lau and Tang's converging-diverging duct ${ }^{9}$, with plane wave excitation. For each plot, $r_{1}=0.5 \mathrm{~m}, r_{2}=0.2 r_{1}$, and $L=2 r_{1}$. (a) $k_{r}=3 / \pi$, $L_{c}=L_{d}=0.2309 \mathrm{~m} ;$ (b) $k_{r}=1, L_{c}=L_{d}=0.2309 \mathrm{~m} ;$ (c) $k_{r}=4 / \pi, L_{c}=L_{d}=0.6928 \mathrm{~m}$. 
Kirby, JASA

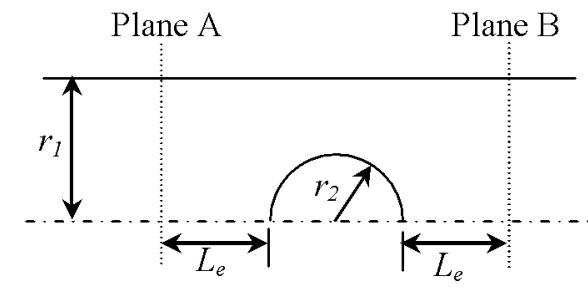

Figure 10. Geometry of circular cylinder. 
Kirby, JASA

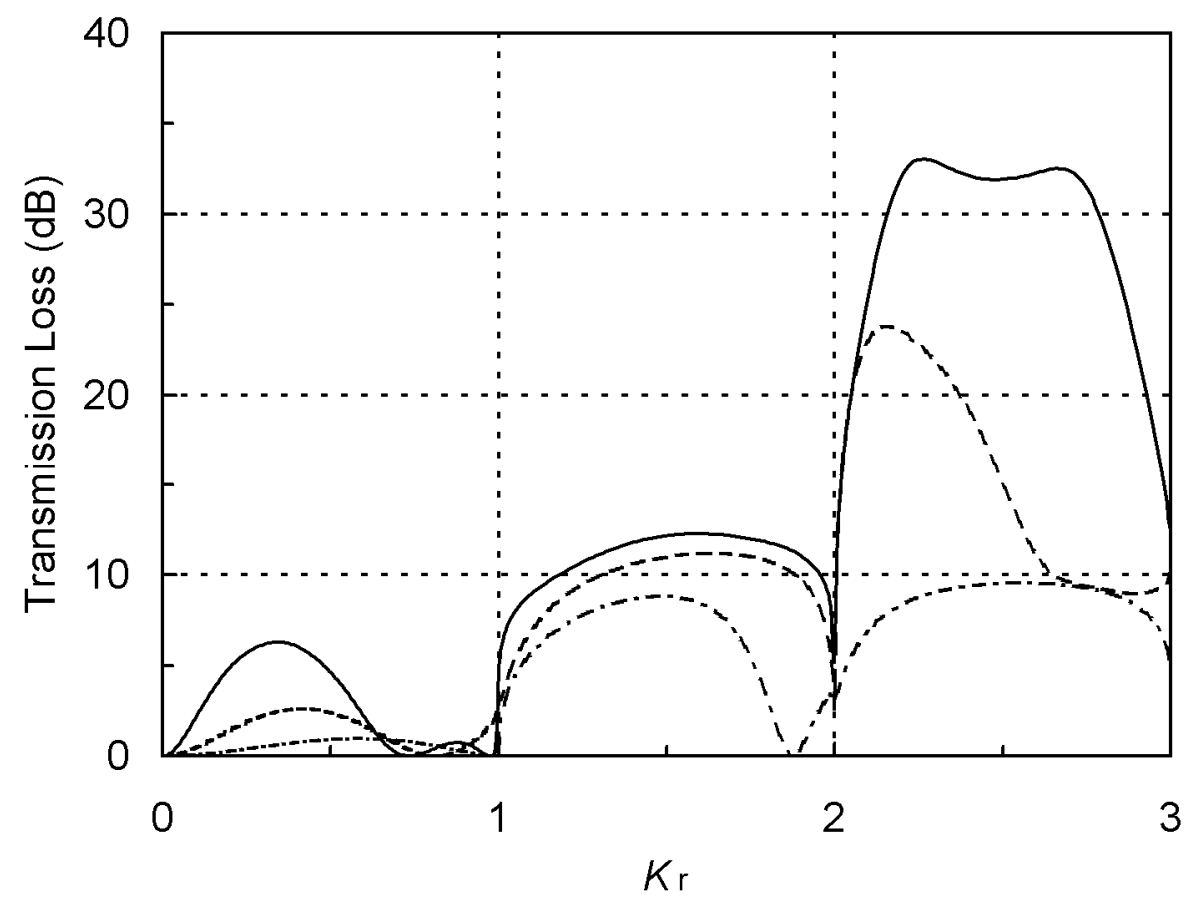

Figure 11. Hybrid method (MM) predictions for circular cylinder with multi-mode (EMED) excitation. For each plot, $r_{1}=0.5 \mathrm{~m}$ and $L_{e}=0.5 r_{2}$. (a) $\longrightarrow, r_{2}=0.8 r_{1} ;$ (b); $-\longrightarrow$, $r_{2}=0.6 r_{1} ;(\mathrm{c})----, r_{2}=0.4 r_{1}$. 